\title{
Balance Deficits and Functional Disability in Cancer Survivors Exposed to Neurotoxic Cancer Treatments
}

\author{
J. Matt McCrary, PhDa; David Goldstein, MBBS, FRCP, FRACPa,b; Terry Trinh, PhDa; Hannah C. Timmins, BSc(Hons)c; \\ Tiffany Li, MBiostatc; Jasmine Menant, PhD d; Michael Friedlander, MBChB(Hons), PhD, MRCP, FRACPa,b; \\ Craig R. Lewis, MBBS, MMed, FRACPa,b; Mark Hertzberg, MBBS, PhD, FRACP, FRCPA a,b; \\ Siobhan O'Neill, MBBS, FRACPb; Tracy King, RN, MNe,f; Annmarie Bosco, MBBS, FRACP, FRCPA ${ }^{b, g}$; \\ Michelle Harrison, MBBS(Hons), FRACPe,h; and Susanna B. Park, PhDa,c
}

\begin{abstract}
Background: Chemotherapy-induced peripheral neuropathy (CIPN) persists after treatment in up to $40 \%$ of cancer survivors and has been linked with increased balance deficits, disabilities, and fall occurrences. This study aimed to comprehensively assess the links between CIPN, balance deficits, and functional disability and to inform the development of clinical screening tools for patients at risk of these events. Patients and Methods: A total of 190 cancer survivors exposed to neurotoxic chemotherapies (age, $57 \pm 13$ years; average time from completion of neurotoxic therapy, $12 \pm 11$ months) attended a neurology research clinic for a single cross-sectional assessment of patient-reported and objective CIPN, standing balance in 4 conditions of increasing difficulty, and functional disability. Results: Most patients (68\%) reported CIPN symptoms at assessment. Symptomatic patients displayed increased functional disability $(\mathrm{F}=39.4 ; P<.001)$ and balance deficits $(\mathrm{F}=34.5 ; P<.001)$, with degree of balance impairments consistent with a healthy elderly population (age $\geq 65$ years) reporting multiple falls over the subsequent year. Increasing CIPN severity correlated with increasing functional disability (clinically assessed $R^{2}=0.46$; patient-reported $R^{2}=0.49 ; P<.001$ ) and balance deficits (clinically assessed $R^{2}=0.41$; patient-reported $R^{2}=0.30 ; P<.001$ ). A 5 -factor model of key independent correlates-patient-reported numbness/tingling, weakness, and balance deficit; age; and vibration perception-was strongly linked to balance deficits $\left(R^{2}=0.46 ; P<.001\right)$ and functional disability $\left(R^{2}=0.56 ; P<.001\right)$. Conclusions: This study confirms links between increasing CIPN severity and increasing balance deficits and functional disability using comprehensive CIPN assessment methodology. The extent of balance deficits in patients with CIPN underscores the functional consequences of neurotoxicity. A 5 -factor model provides a foundation for clinical screening tools to assess balance deficits and functional disability in patients exposed to neurotoxic chemotherapies.
\end{abstract}

J Natl Compr Canc Netw 2019;17(8):949-955 doi: $10.6004 /$ jnccn.2019.7290

\footnotetext{
aPrince of Wales Clinical School, University of New South Wales, Kensington; ${ }^{b}$ Prince of Wales Hospital, Randwick; ${ }^{\circ}$ Brain and Mind Centre, The University of Sydney, Camperdown; 'Neuroscience Research Australia, Randwick; ${ }^{\text {Royal }}$

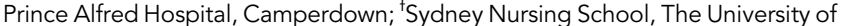
Sydney, Camperdown; ${ }^{9}$ School of Medical Sciences, University of New South Wales, Kensington; and ' hThe Chris O'Brien Lifehouse, Camperdown, Australia.
}

\section{Background}

There are now millions of cancer survivors worldwide, ${ }^{1}$ with this increasing population requiring appropriate strategies and interventions to support quality of life and function and minimize long-term adverse effects of cancer and its treatment. Chemotherapy-induced peripheral neuropathy (CIPN) is a significant dose-limiting adverse effect of cancer treatment and is estimated to impact $68 \%$ of patients treated with neurotoxic chemotherapies, leading to functional disability and permanent symptoms in up to $40 \%$ of cancer survivors. ${ }^{2,3}$ CIPN is a predominantly sensory neuropathy presenting as numbness/tingling in the hands and feet, although it may be accompanied by myalgia, muscle cramps, and distal weakness. ${ }^{4} \mathrm{CIPN}$ has been associated with gait, balance, and fine motor deficits ${ }^{4-8}$; more severe CIPN symptoms are linked to greater functional disability and balance deficits ${ }^{6,9}$ and a higher incidence of falls. ${ }^{9}$

Despite the significant effects of CIPN on balance and functional disability, investigations of its functional impact remain complicated by the lack of a gold standard assessment. Although no consensus has been reached on the best CIPN assessment, ${ }^{10}$ a combination of patientreported outcomes and clinical or objective measures provides the most comprehensive approach. ${ }^{11}$ For clinical use, a recent Delphi survey showed that any CIPN assessment with $\geq 6$ items is unlikely to be feasible. ${ }^{10}$ The development of targeted assessments to facilitate clinical screening of patients with CIPN at risk of balance deficits and functional disability has also been identified as an area of research need. ${ }^{12}$ Currently, however, no study has investigated the links between CIPN severity, balance deficits, and functional disability using a comprehensive assessment approach. Pathways to efficient clinical

See JNCCN.org for supplemental online content. 
assessment of survivors at risk for functional balance impairments and functional disability have also yet to be investigated.

This study aims to address these knowledge gaps through investigating the links between CIPN symptom severity, balance deficits, and functional disability using a combination of validated clinical and patient-reported outcome assessments, and identifying individual demographic and assessment items with the strongest independent links to balance deficits to provide a foundation for streamlined clinical screening of patients at risk for balance deficits and functional disability.

\section{Patients and Methods}

\section{Patients}

Our study cohort included cancer survivors 3 months to 5 years posttreatment with known neurotoxic cancer treatments (oxaliplatin, cisplatin, docetaxel, paclitaxel, nab-paclitaxel, thalidomide, vincristine, bortezomib, lenalidomide, vinblastine, vinorelbine $)^{4}$ who underwent comprehensive testing including a standing balance assessment in ongoing observational studies (IN FOCUS study; www.infocusstudy.org.au) between June 2016 and May 2018. Patients with a confirmed diabetes diagnosis were included if no neuropathy symptoms were present before commencing neurotoxic cancer treatment. This study was approved by the Human Research Ethics Committees of South Eastern Sydney Local Health District and Sydney Local Health District (Royal Price Alfred Hospital zone) and was conducted according to the Declaration of Helsinki. All patients provided written informed consent before study participation.

\section{Balance Assessment}

Postural sway, a quantification of postural instability, ${ }^{13}$ was measured using a Swaymeter, a valid, reliable, and portable method of quantifying postural sway without need for a force plate (Neuroscience Research Australia [NeuRA]). ${ }^{14,15}$ Sturnieks et al ${ }^{14}$ published a full description of the device and procedures, including diagrams.

Using standing balance assessment procedures from the FallScreen protocol, ${ }^{15}$ the Swaymeter measured displacements of the body using a $40-\mathrm{cm}$ rod attached to the patient's waist by a firm belt and extended behind in the horizontal plane. A stylus mounted vertically at the end of the rod recorded the movements of the center of mass (total path length; millimeters) on an iPad using the PPA Sway Path app (NeuRA). Patients were instructed to find a comfortable, stable stance and stand "as still as possible without talking" for 30 seconds while barefoot in 4 conditions of increasing challenge: standing on the floor with (1) eyes open and (2) eyes closed, and standing on foam with (3) eyes open and (4) eyes closed.
A sumscore of the total path length from all 4 tasks was used as the primary balance outcome for analysis, given prior studies demonstrating better representation of overall balance impairments by composite balance scores versus individual tests. ${ }^{16,17}$ Patients unable to complete any tasks due to excess instability were given a score equivalent to 3 standard deviations above the mean for the given task, according to prior convention. ${ }^{18}$ Data were statistically compared with normative data from healthy and elderly populations, which used the same equipment and test protocol. ${ }^{18-20}$

\section{Neuropathy Assessment Tools}

\section{Clinical CIPN Severity}

Clinical CIPN severity was graded using the Total Neuropathy Score clinical version (TNSc), ${ }^{21}$ which includes a clinical assessment of symptoms, muscle weakness and pinprick sensibility, vibration sensibility, tendon reflexes, and strength. A higher score on the TNSc indicates greater neuropathy severity (range, 0-24).

\section{Patient-Reported CIPN Symptoms}

Patient-reported neuropathy symptoms were assessed using the EORTC QLQ-CIPN20 questionnaire (CIPN20), ${ }^{22}$ a 20-item questionnaire related to sensory, motor, and autonomic symptoms of neuropathy. A higher score on this questionnaire represents increased symptom burden, with each item scored from 1 (not at all) to 4 (very much), and the total questionnaire is converted to a score from 0 to 100 .

Additionally, a "CIPN symptom index" comprising the first 4 items of the CIPN20 was used to measure the extent of sensory symptoms and define asymptomatic versus symptomatic patients. The first 4 items of the CIPN20 refer to the severity of numbness/tingling in the hands and feet. CIPN symptom index scores total 4 to 16 points and were classified as either no symptoms/ asymptomatic (4 points) or symptomatic ( $\geq 5$ points: mild symptoms, 5-8 points; moderate symptoms, 9-12 points; or severe symptoms, $13-16$ points).

\section{Functional Disability Assessment}

The CIPN Rasch-built Overall Disability Scale (CIPNR-ODS), ${ }^{23}$ a disease-specific 28-item patient-reported outcome questionnaire designed to assess general disability in a CIPN population, was used to evaluate activity limitations and participation restrictions. The CIPNR-ODS is scored on a scale of 0 to 100 , with a lower score indicating greater functional disability.

\section{Data Analysis}

Independent samples $t$-tests and chi-square tests were used to compare demographic variables between asymptomatic (CIPN symptom index of 4; control group) and 
symptomatic patients (CIPN symptom index $\geq 5$ ). Independent samples $t$-tests were also used to compare postural sway sumscores between data from our study cohort and data from healthy $\left(n=30\right.$ and $n=43,{ }^{18}$ respectively) and elderly populations $\left(n=98^{19}\right.$ and $n=341,{ }^{20}$ respectively). One-way analysis of covariances (ANCOVAs) were used to examine differences in postural sway between the 2 groups while controlling for demographic variables that varied significantly. Significance was set at $\alpha=0.05$ for $t$-tests, chi-square tests, and ANCOVAs described in the prior sections.

Five bivariate linear regression models were used to determine relationships for the entire patient cohort between postural sway sumscore (dependent variable) and clinical and patient-reported CIPN symptom severity (CIPN20 and TNSc) and functional disability (independent variables), and functional disability (dependent variable) and clinical and patient-reported CIPN symptom severity (CIPN20 and TNSc) (independent variables). These models were adjusted for increasing age to control for its known effects on postural sway and functional disability. ${ }^{24,25}$ Significance was set at $\alpha=0.05$ for the 5 models.

Bivariate linear regression models were also used to investigate the relationships between postural sway sumscore (dependent variable) and age, medically confirmed diabetes diagnosis, and each individual item of the TNSc and CIPN20 assessments (independent variables). CIPN20 items 1 through 4 (CIPN symptom index), 5 and 6 (shooting and burning pains in hands and feet), and 7 and 8 (cramps in hands and feet) were analyzed as composite items. Age, TNSc item 1 (severity of numbness/tingling), and CIPN symptom index were analyzed as continuous or ordinal variables because of links between age and CIPN severity and balance deficits. ${ }^{6,24,25}$ All other variables were dichotomous (presence or absence of the symptom or clinical abnormality). Diabetes diagnosis was included as an independent variable to account for any additive impact it might have on balance deficits appearing after neurotoxic cancer treatment; diabetes is independently associated with balance deficits, ${ }^{26}$ although its impact on CIPN and related functional impairments is presently unclear. ${ }^{27,28}$ The Bonferroni-Holm correction for multiple comparisons was applied to all bivariate regression models, with the adjusted $\alpha$-level used to determine significance..$^{29}$

Using variables significantly associated with postural sway sumscore from the bivariate analyses $(P<$ Bonferroni-Holm-adjusted $\alpha$ ), multiple linear regression models were used to inform the development of clinical screening tools for patients at risk of balance deficits and functional disability. Two multiple regression models were created. The first was a stepwise multiple linear regression model used to determine significant independent variables impacting postural sway. The second was a multiple regression model that examined the relationships between patient-reported functional disability and the significant independent variables from the stepwise model. Significance was set at $\alpha=0.05$ for the 2 multiple linear regression models.

To determine the additive effect of the significant independent variables identified in the stepwise model, each symptom or clinical abnormality represented by the independent variables was classified as a risk factor. Continuous variables were converted into binary forms as appropriate, such that all independent variables were given a binary risk factor classification. Bivariate linear regression models were used to determine the relationships between the number of risk factors presented (independent variable) and postural sway sumscore and functional disability (dependent variables). Additionally, a 1-way analysis of variance (ANOVA) with Bonferroni post hoc test was used to evaluate discrete differences between postural sway sumscore and CIPN-R-ODS score given the number of risk factors presented. Significance was set at $\alpha=0.05$ for bivariate linear regression models, ANOVAs, and post hoc tests associated with the risk factor classifications.

All analyses were conducted using SPSS Statistics, version 24.0 (IBM Corporation).

\section{Results}

\section{Patient Characteristics}

The study cohort included 190 patients treated with potentially neurotoxic chemotherapies. Most patients were treated for breast cancer $(n=75 ; 39.5 \%)$, colorectal cancer $(n=29 ; 15.3 \%)$, or lymphoma $(n=20 ; 10.5 \%)$ (Table 1). Other cancer types represented included myeloma and gynecologic, testicular, gastrointestinal, pancreatic, head and neck, lung, cardiac, liver, prostate, and urothelial cancers. The most commonly received types of neurotoxic chemotherapy were taxanes $(n=103$; $54.2 \%$ ), followed by the platinum-based agents oxaliplatin or cisplatin $(n=54 ; 28.4 \%)$.

Most patients reported numbness/tingling at the time of testing $(\mathrm{n}=129 ; 67.9 \%$; CIPN symptom index score $\geq 5$, with $61.2 \%$ having mild symptoms, $28.7 \%$ moderate symptoms, and $10.1 \%$ severe symptoms). Clinical evidence of CIPN was identified in 140 patients (73.7\%; TNSc score $\geq 2$ ).

Symptomatic patients were significantly older ( $\mathrm{F}=25.6 ; P<.001)$, reported more functional disability $(\mathrm{F}=39.4 ; P<.001)$, and showed significantly increased postural sway sumscore $(\mathrm{F}=34.5 ; P<.001)$. Additionally, a significantly greater proportion of symptomatic patients were survivors of colorectal cancer and had 


\section{Table 1. Patient Demographics}

\begin{tabular}{|c|c|c|}
\hline & $\begin{array}{l}\text { No Symptoms } \\
(\mathbf{N}=61)\end{array}$ & $\begin{array}{c}\text { Symptoms } \\
(\mathrm{N}=129)\end{array}$ \\
\hline Age, $y^{a}( \pm S D)$ & $50.2(13.8)$ & $59.7(11.5)$ \\
\hline Sex (male:female) & $17: 44$ & $48: 81$ \\
\hline Months since treatment $( \pm \mathrm{SD}$ ) & $13.4(13.3)$ & $11.0(10.1)$ \\
\hline Diabetes diagnosis, $\mathrm{n}$ & 4 & 14 \\
\hline Disability ${ }^{a}$ (CIPN-R-ODS) ( \pm SD) & $94.8(7.2)$ & $83.9(12.8)$ \\
\hline Clinical CIPNa (TNSc) ( \pm SD) & $1.7(1.6)$ & $5.1(3.2)$ \\
\hline Patient-reported CIPN ${ }^{a}(C I P N 20)$ ( \pm SD) & $2.6(3.9)$ & $18.6(14.6)$ \\
\hline \multicolumn{3}{|l|}{ Postural sway path length, mm ( \pm SD) } \\
\hline Eyes open, floor ${ }^{\mathrm{a}}$ & $90.0(38.4)$ & $118.1(83.3)$ \\
\hline Eyes closed, floor ${ }^{a}$ & $112.8(48.2)$ & $192.1(110.7)$ \\
\hline Eyes open, foam ${ }^{a}$ & $145.6(45.1)$ & $203.1(102.6)$ \\
\hline Eyes closed, foam & $313.5(118.6)$ & $460.4(196.0)$ \\
\hline Sumscore ${ }^{a}$ & $661.8(187.7)$ & $973.7(392.9)$ \\
\hline \multicolumn{3}{|c|}{ Severity of numbness/tingling on CIPN symptom index, $n(\%)$} \\
\hline None & $61(100)$ & - \\
\hline Mild & - & $79(61.2)$ \\
\hline Moderate & - & $37(28.7)$ \\
\hline Severe & - & $13(10.1)$ \\
\hline \multicolumn{3}{|l|}{ Chemotherapy type, ${ }^{a}$ (\%) } \\
\hline Paclitaxel & $22(36.1)$ & $41(34.5)$ \\
\hline Oxaliplatin & $3(4.9)$ & $35(29.4)$ \\
\hline Carboplatin/Paclitaxel & $9(14.8)$ & $16(13.4)$ \\
\hline Vincristine & $7(11.5)$ & $12(10.1)$ \\
\hline Cisplatin & $7(11.5)$ & $9(7.6)$ \\
\hline Docetaxel & $7(11.7)$ & $6(5.0)$ \\
\hline Bortezomib & $2(3.3)$ & $5(4.2)$ \\
\hline Thalidomide & $1(1.6)$ & $2(1.7)$ \\
\hline Carboplatin & $1(1.6)$ & $1(0.8)$ \\
\hline nab-Paclitaxel & $1(1.6)$ & - \\
\hline Carboplatin/Docetaxel & $1(1.6)$ & - \\
\hline Bortezomib/Thalidomide & - & $2(1.7)$ \\
\hline \multicolumn{3}{|l|}{ Cancer type, ${ }^{a}$ n (\%) } \\
\hline Breast & $29(47.5)$ & $46(38.7)$ \\
\hline Colorectal & $2(3.3)$ & $27(22.7)$ \\
\hline Lymphoma & $7(11.4)$ & $13(10.9)$ \\
\hline Myeloma & $3(4.9)$ & $9(7.6)$ \\
\hline Ovarian & $6(9.8)$ & $6(5.0)$ \\
\hline Other & $14(23.0)$ & $28(23.5)$ \\
\hline
\end{tabular}

Abbreviations: CIPN, chemotherapy-induced peripheral neuropathy; CIPN20, EORTC QLQ-CIPN20 questionnaire; CIPN-R-ODS, CIPN Rasch-built Overall Disability Scale; TNSc, Total Neuropathy Score clinical version.

asignificant differences across groups $(P<.01)$.

received oxaliplatin treatment $(P<.02)$. Differences in postural sway and functional disability between symptomatic and asymptomatic patients remained significant when adjusting for age, cancer type, and chemotherapy type $(\mathrm{F}=12.8 ; P<.001)$. Results and demographics stratified by symptom status are shown in Table 1.

Both symptomatic and asymptomatic patients showed higher postural sway sumscore values compared with age-matched normative values $(P<.001$; healthy participants aged $40-59$ years: $414.3 \pm 90.1 \mathrm{~mm}$; aged 60-64 years: $451.9 \pm 99.3 \mathrm{~mm}$ ). ${ }^{18}$ Postural sway sumscore in patients with CIPN symptoms was greater than $(P<.001)$ or similar to $(P=.37)$ values associated with multiple falls over a 1-year period in prospective studies of elderly populations $\left(700 \pm 308 \mathrm{~mm}^{19}\right.$ and $1,016 \pm$ $472 \mathrm{~mm},{ }^{20}$ respectively).

\section{CIPN Severity and Balance and Functional Disability: Bivariate Regression Models}

Increasing CIPN severity, as assessed using both patientreported and clinical methods, and patient-reported functional disability were significantly associated with increasing postural sway (age-adjusted CIPN20, $\mathrm{R}^{2}=0.17 ; P<.001$ and TNSc, $\mathrm{R}^{2}=0.23 ; P<.001 ; \mathrm{CIPN}$ $\mathrm{R}$-ODS, $\mathrm{R}^{2}=0.24 ; P<.001$ ) (Figure 1 ). Increasing severity of patient-reported and clinical CIPN was also significantly associated with increasing functional disability (age-adjusted CIPN20, $\mathrm{R}^{2}=0.13 ; P<.001$; TNSc, $\left.\mathrm{R}^{2}=0.06 ; P<.001\right)$.

\section{Significant Independent Variables Impacting}

Postural Sway: Multiple Regression Models

Bivariate models identified 18 independent variables associated with increasing postural sway $(0.24<R<0.51$; $P \leq .001$; supplemental eTable 1 , available with this article at JNCCN.org). Stepwise linear regression analysis produced a model including 5 significant independent variables impacting postural sway: patient-reported balance or mobility deficit, numbness/tingling in hands or feet (any severity), and leg weakness "leading to difficulty climbing stairs or getting up out of a chair"; abnormal vibration perception; and older age $\left(\geq 65\right.$ years $\left.^{18}\right)\left(\mathrm{R}^{2}=0.46 ; P<.001\right.$; Table 2$)$. A model with these 5 variables was also associated with patientreported functional disability $\left(\mathrm{R}^{2}=0.56 ; P<.001\right.$; supplemental eTable 2).

When each of the 5 independent variables was classified as a binary risk factor, the number of risk factors presented were strongly associated with both postural sway sumscore $\left(\mathrm{R}^{2}=0.45 ; P<.001\right)$ and patientreported functional disability $\left(\mathrm{R}^{2}=0.48 ; P<.001\right)$ (Figure 2). Continuous variables, such as CIPN symptom index and age, were dichotomized accordingly (eg, presence or absence of any patient-reported numbness and/or tingling; age $<65$ or $\geq 65$ years). ${ }^{18}$ 

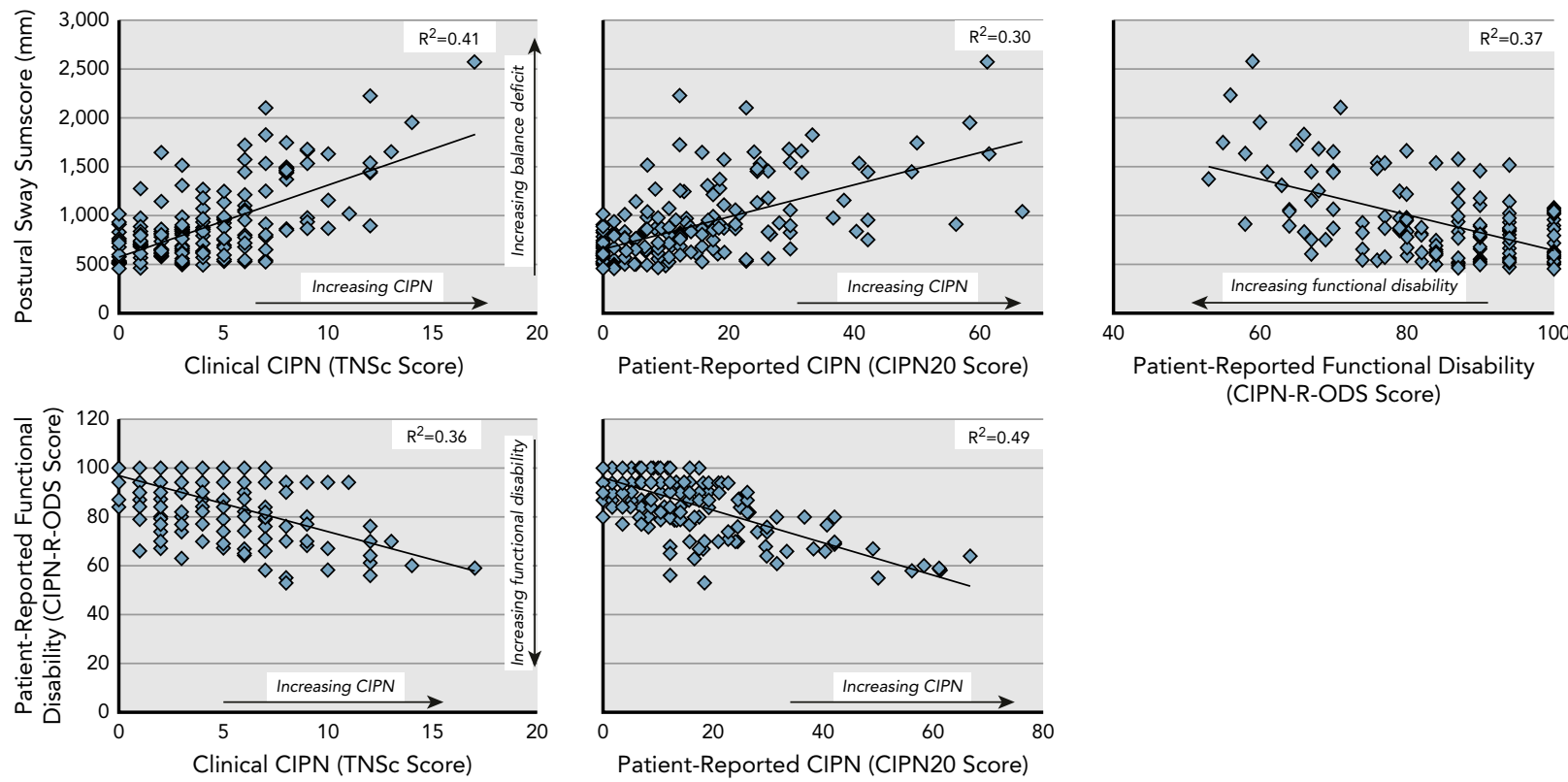

(CIPN-R-ODS Score)

Figure 1. Scatterplots describing linear correlations between postural sway and clinical CIPN (TNSc score), patient-reported CIPN (CIPN20 score), and patient-reported disability (CIPN-R-ODS). All displayed relationships are significant $(P<.001)$. All $R^{2}$ values are unadjusted.

Abbreviations: CIPN, chemotherapy-induced peripheral neuropathy; CIPN2O, EORTC OLQ-CIPN20 questionnaire; CIPN-R-ODS, CIPN Rasch-built Overall Disability Scale; TNSc, Total Neuropathy Score clinical version.

\section{Discussion}

This study, using comprehensive CIPN assessment methodology and a large cohort, provides strong evidence of increased balance deficits and functional disability in cancer survivors with CIPN symptoms, and shows that greater CIPN symptom severity is associated with increased balance deficits and functional disability. Postural sway was increased compared with agematched normative values ${ }^{18}$ in both symptomatic and asymptomatic patients, consistent with prior research noting general functional deficits in cancer survivors. ${ }^{30,31}$ Postural sway results in symptomatic patients were also similar to or greater than values associated with elderly patients reporting multiple falls, ${ }^{19,20}$ underscoring the potential consequences of the degree of balance impairments in survivors with CIPN. Additionally, this investigation presents key independent factors impacting balance performance (patient-reported balance deficits, numbness/tingling, and leg weakness; abnormal vibration perception; age), providing an evidence-based foundation for the development of a quick clinical screening tool for patients at risk of balance deficits and functional disability.

Using comprehensive CIPN assessment methodology, this study provides additional weight to previous studies that similarly show links between CIPN symptoms and balance deficits. These prior studies have identified these CIPN-related balance deficits, both compared with asymptomatic survivors exposed to neurotoxic cancer treatments ${ }^{8,9}$ and with respect to increasing CIPN severity. ${ }^{6}$ Links between increased postural sway and increased functional disability

\begin{tabular}{|c|c|c|c|c|}
\hline & Assessment Item & $\mathbf{R}$ & $\beta$ & $P$ Value \\
\hline Full model & & 0.68 & & $<.001$ \\
\hline Patient-reported balance/mobility deficit & CIPN20 \#9 & 0.51 & 0.24 & $<.001$ \\
\hline Abnormal vibration perception & TNSc \#4 & 0.60 & 0.22 & $<.001$ \\
\hline Patient-reported numbness/tingling & CIPN20 \#1-4 & 0.64 & 0.20 & .004 \\
\hline Age ( $\geq 65$ years) & & 0.66 & 0.18 & .004 \\
\hline Patient-reported leg weakness & CIPN20 \#15 & 0.68 & 0.16 & .014 \\
\hline
\end{tabular}

Abbreviations: CIPN, chemotherapy-induced peripheral neuropathy; CIPN2O, EORTC QLQ-CIPN20 questionnaire; TNSc, total neuropathy score.

a $P$ values correspond to the significance of $\beta$ weights for each significant correlate. 
A

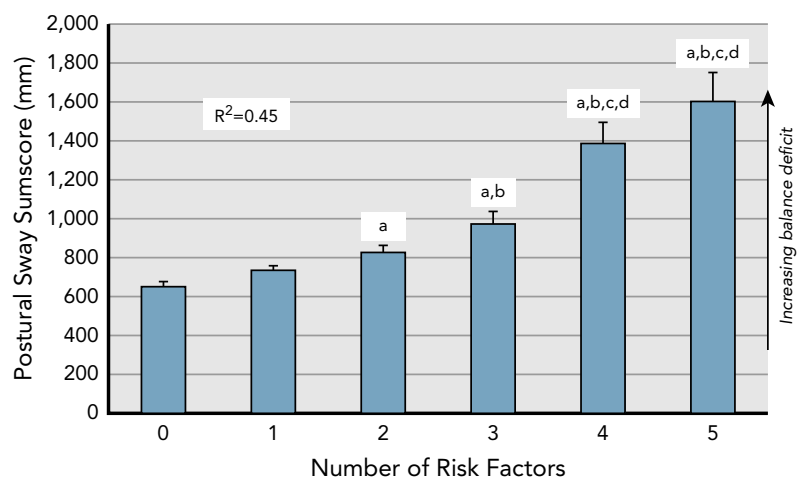

B

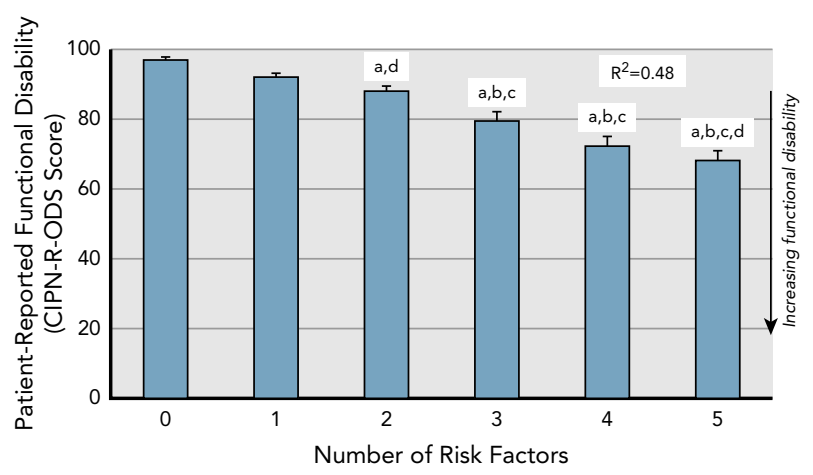

Figure 2. (A) Postural sway and (B) functional disability with respect to the number of clinical risk factors for balance impairment as identified in Table 2. All results presented as mean $+\mathrm{SE}$. ANOVA main effects: postural sway $(F=38.1 ; P<.001)$; disability $(F=34.6 ; P<.001)$.

"a" indicates significant difference from 0 risk factors $(P<.04)$; " $b$ " indicates significant difference from 1 risk factor $(P<.01)$; " $c$ " indicates significant difference from 2 risk factors $(P<.001)$; and " $\mathrm{d}$ " indicates significant difference from 3 risk factors $(P<.001)$.

Abbreviations: ANOVA, analysis of variance; CIPN, chemotherapy-induced peripheral neuropathy; CIPN-R-ODS, CIPN Rasch-built Overall Disability Scale.

mirror prior research in $\mathrm{CIPN}^{9}$ and cancer survivors demonstrating that balance control is a determinant of patient-reported functional disability. ${ }^{32}$

In the 5-factor model, independent links between postural sway and patient reports of balance deficits and numbness/tingling highlight the importance of patientreported outcomes in CIPN assessments ${ }^{11}$ and provide further evidence that numbness and tingling are the most critical CIPN symptoms influencing functional impairments. ${ }^{9,21}$ Furthermore, vibration perception has been correlated with comprehensive and neurophysiologic CIPN assessments ${ }^{11,33}$ and increased postural sway in elderly populations. ${ }^{34}$ Our model further shows that vibration perception may provide a quick, objective assessment linked to neurophysiologic and postural dysfunction. Older age and muscle weakness are both known contributors to balance deficits ${ }^{35}$ and were expected independent factors impacting postural sway in this study.
A limitation of this study was that the validity, sensitivity, reliability, and feasibility of using the 5 -factor model in clinical screening for balance deficits and functional disability in CIPN are not addressed. Further research is required to address these domains. However, the strong relationships between an increasing number of risk factors and increasing postural sway and functional disability suggest that the model may show clinical utility. Furthermore, inclusion of the preferred combination of clinical and patient-reported assessment strategies ${ }^{11}$ within a small number of items ${ }^{10}$ is an indicator of the prospective feasibility of the 5 -factor model. The clinical applications of vibration testing from our model are potentially limited by the use of specialized semiquantitative methods (Rydel-Seiffer tuning fork), although the broad utility of vibration testing described already and incorporating a variety of techniques ${ }^{11,33,34}$ temper these concerns. Additionally, this study is limited by its cross-sectional design, and further prospective research is required to investigate causal relationships between CIPN and balance and functional disability.

\section{Conclusions}

Using comprehensive CIPN assessment methodology, our findings confirm that increased CIPN severity is linked to increased balance deficits and functional disability. Postural sway values in patients with symptomatic CIPN were consistent with those of elderly patients reporting multiple falls, emphasizing the potential consequences of functional impairments related to CIPN. A 5-factor model (patient-reported balance deficits, numbness/tingling, and leg weakness; abnormal vibration perception; and age) showed strong correlations with balance deficits and functional disability, providing a foundation for the development of broadly applicable clinical screening tools for patients with CIPN at risk of functional impairments.

Submitted December 4, 2018; accepted for publication February 25, 2019.

Author contributions: Study concept and design: McCrary, Goldstein, Park. Data acquisition: McCrary, Trinh, Timmins, Li. Quality control of data and algorithms: McCrary, Menant. Data analysis and interpretation: McCrary, Goldstein, Menant, Park. Statistical analysis: McCrary. Manuscript preparation: McCrary. Manuscript editing and review: All authors.

Disclosures: The authors have disclosed that they have not received any financial considerations from any person or organization to support the preparation, analysis, results, or discussion of this article.

Funding: This study was supported by a Cancer Institute NSW Program Grant (14/TPG/1-05; Goldstein) and a National Health and Medical Research Council of Australia (NHMRC) Project Grant (1080521; Park). Dr. Park is supported by a NHMRC Career Development Fellowship (1148595).

Correspondence: Susanna B. Park, PhD, Brain and Mind Centre, The University of Sydney, 94 Mallett Street, Camperdown, NSW 2050, Australia. Email: susanna.park@sydney.edu.au 


\section{References}

1. Miller KD, Siegel RL, Lin CC, et al. Cancer treatment and survivorship statistics, 2016. CA Cancer J Clin 2016;66:271-289.

2. Wolf $S$, Barton $D$, Kottschade $L$, et al. Chemotherapy-induced peripheral neuropathy: prevention and treatment strategies. Eur J Cancer 2008;44: 1507-1515.

3. Seretny M, Currie GL, Sena ES, et al. Incidence, prevalence, and predictors of chemotherapy-induced peripheral neuropathy: a systematic review and meta-analysis. Pain 2014;155:2461-2470.

4. Park SB, Goldstein D, Krishnan AV, et al. Chemotherapy-induced peripheral neurotoxicity: a critical analysis. CA Cancer J Clin 2013;63: 419-437.

5. Kneis S, Wehrle A, Freyler K, et al. Balance impairments and neuromuscular changes in breast cancer patients with chemotherapy-induced peripheral neuropathy. Clin Neurophysiol 2016;127:1481-1490.

6. Monfort SM, Pan X, Patrick R, et al. Gait, balance, and patient-reported outcomes during taxane-based chemotherapy in early-stage breast cancer patients. Breast Cancer Res Treat 2017;164:69-77.

7. Wampler MA, Topp KS, Miaskowski C, et al. Quantitative and clinical description of postural instability in women with breast cancer treated with taxane chemotherapy. Arch Phys Med Rehabil 2007;88: 1002-1008

8. Miaskowski C, Mastick J, Paul SM, et al. Chemotherapy-induced neuropathy in cancer survivors. J Pain Symptom Manage 2017;54 204-218.

9. Winters-Stone KM, Horak F, Jacobs PG, et al. Falls, functioning, and disability among women with persistent symptoms of chemotherapyinduced peripheral neuropathy. J Clin Oncol 2017;35:2604-2612.

10. McCrary JM, Goldstein D, Boyle F, et al. Optimal clinical assessment strategies for chemotherapy-induced peripheral neuropathy (CIPN): a systematic review and Delphi survey. Support Care Cancer 2017;25: 3485-3493.

11. Alberti P, Rossi E, Cornblath DR, et al. Physician-assessed and patientreported outcome measures in chemotherapy-induced sensory peripheral neurotoxicity: two sides of the same coin. Ann Oncol 2014;25: 257-264.

12. Stout NL. Expanding the perspective on chemotherapy-induced peripheral neuropathy management. J Clin Oncol 2017;35:2593-2594.

13. Maki BE, Holliday PJ, Topper AK. A prospective study of postural balance and risk of falling in an ambulatory and independent elderly population. J Gerontol 1994;49:M72-84.

14. Sturnieks DL, Arnold R, Lord SR. Validity and reliability of the Swaymeter device for measuring postural sway. BMC Geriatr 2011;11:63.

15. Lord SR, Menz HB, Tiedemann A. A physiological profile approach to falls risk assessment and prevention. Phys Ther 2003;83:237-252.

16. Panzer VP, Wakefield DB, Hall CB, et al. Mobility assessment: sensitivity and specificity of measurement sets in older adults. Arch Phys Med Rehabil 2011;92:905-912

17. Wallmann HW. Comparison of elderly nonfallers and fallers on performance measures of functional reach, sensory organization, and limits of stability. J Gerontol A Biol Sci Med Sci 2001;56:M580-583.
18. Lord SR, Ward JA. Age-associated differences in sensori-motor function and balance in community dwelling women. Age Ageing 1994;23:452-460.

19. Lord SR, Clark RD, Webster IW. Physiological factors associated with falls in an elderly population. J Am Geriatr Soc 1991;39:1194-1200.

20. Lord SR, Ward JA, Williams $P$, et al. Physiological factors associated with falls in older community-dwelling women. J Am Geriatr Soc 1994;42:1110-1117.

21. Cornblath DR, Chaudhry V, Carter K, et al. Total neuropathy score: validation and reliability study. Neurology 1999;53:1660-1664.

22. Postma TJ, Aaronson NK, Heimans JJ, et al. The development of an EORTC quality of life questionnaire to assess chemotherapy-induced peripheral neuropathy: the QLQ-CIPN20. Eur J Cancer 2005;41: 1135-1139.

23. Binda D, Vanhoutte EK, Cavaletti G, et al. Rasch-built Overall Disability Scale for patients with chemotherapy-induced peripheral neuropathy (CIPN-R-ODS). Eur J Cancer 2013:49:2910-2918.

24. Tiedemann A, Shimada $H$, Sherrington $C$, et al. The comparative ability of eight functional mobility tests for predicting falls in community-dwelling older people. Age Ageing 2008;37:430-435.

25. Sheldon JH. The effect of age on the control of sway. Gerontol Clin (Basel) 1963:5:129-138.

26. Schwartz AV, Hillier TA, Sellmeyer DE, et al. Older women with diabetes have a higher risk of falls: a prospective study. Diabetes Care 2002;25: 1749-1754.

27. Brouwers EE, Huitema AD, Boogerd W, et al. Persistent neuropathy after treatment with cisplatin and oxaliplatin. Acta Oncol 2009;48: 832-841.

28. Kus T, Aktas G, Kalender ME, et al. Taxane-induced peripheral sensoria neuropathy in cancer patients is associated with duration of diabetes mellitus: a single-center retrospective study. Support Care Cancer 2016 24:1175-1179.

29. Ludbrook J. Multiple comparison procedures updated. Clin Exp Pharmacol Physiol 1998;25:1032-1037.

30. Peters E, Mendoza Schulz L, Reuss-Borst M. Quality of life after cancerhow the extent of impairment is influenced by patient characteristics. BMC Cancer 2016;16:787

31. Huang MH, Blackwood J, Godoshian M, et al. Prevalence of self-reported falls, balance or walking problems in older cancer survivors from Surveillance, Epidemiology and End Results-Medicare Health Outcomes Survey. J Geriatr Oncol 2017;8:255-261.

32. Huang MH, Lytle T, Miller KA, et al. History of falls, balance performance, and quality of life in older cancer survivors. Gait Posture 2014 40:451-456.

33. Griffith KA, Dorsey SG, Renn CL, et al. Correspondence between neurophysiological and clinical measurements of chemotherapy-induced peripheral neuropathy: secondary analysis of data from the Cl-PeriNomS study. J Peripher Nerv Syst 2014;19:127-135.

34. Lord SR, Clark RD, Webster IW. Postural stability and associated physiological factors in a population of aged persons. J Gerontol 1991;46:M69-76.

35. Horlings CG, van Engelen BG, Allum JH, et al. A weak balance: the contribution of muscle weakness to postural instability and falls. Nat Clin Pract Neurol 2008;4:504-515. 
Supplemental online content for:

\section{Balance Deficits and Functional Disability in Cancer Survivors Exposed to Neurotoxic Cancer Treatments}

J. Matt McCrary, PhD; David Goldstein, MBBS, FRCP, FRACP; Terry Trinh, PhD; Hannah C. Timmins, BSc(Hons); Tiffany Li, MBiostat; Jasmine Menant, PhD; Michael Friedlander, MBChB(Hons), PhD, MRCP, FRACP; Craig R. Lewis, MBBS, MMed, FRACP; Mark Hertzberg, MBBS, PhD, FRACP, FRCPA; Siobhan O'Neill, MBBS, FRACP; Tracy King, RN, MN; Annmarie Bosco, MBBS, FRACP, FRCPA; Michelle Harrison, MBBS(Hons), FRACP; and Susanna B. Park, PhD

J Natl Compr Canc Netw 2019;17(8):949-955

eTable 1: Relationships Between Postural Sway and Individual Assessment or Demographic Items eTable 2: Multiple Regression Analysis of 5 Key Correlates of Postural Sway Sumscore and Patient-Reported Disability 


\section{eTable 1. Relationships Between Postural Sway and Individual Assessment or Demographic Items}

\begin{tabular}{|c|c|c|c|c|}
\hline & Assessment Item & $\mathbf{R}$ & $P$ Value & $\mathbf{n}^{\mathbf{a}}$ \\
\hline Patient-reported balance/mobility deficit & CIPN20 \#9 & 0.51 & $3.6 \times 10^{-14}$ & 49 \\
\hline Patient-reported numbness/tingling ${ }^{\mathrm{b}}$ & CIPN20 \#1-4 & 0.51 & $8.7 \times 10^{-14}$ & - \\
\hline Patient-reported fine motor deficit (pen) & CIPN20 \#11 & 0.46 & $1.9 \times 10^{-11}$ & 40 \\
\hline Numbness/Tingling (clinical) ${ }^{b}$ & TNSc \#1 & 0.46 & $1.6 \times 10^{-10}$ & - \\
\hline Patient-reported leg weakness & CIPN20 \#15 & 0.44 & $3.0 \times 10^{-10}$ & 47 \\
\hline Abnormal vibration perception & TNSc \#4 & 0.42 & $1.4 \times 10^{-9}$ & 54 \\
\hline $\mathrm{Age}^{\mathrm{b}}$ & - & 0.41 & $5.4 \times 10^{-9}$ & - \\
\hline Patient-reported fine motor deficit (buttons) & CIPN20 \#12 & 0.40 & $1.2 \times 10^{-8}$ & 70 \\
\hline Weakness (clinical) & TNSc \#2 & 0.34 & $2.0 \times 10^{-6}$ & 48 \\
\hline Patient-reported shooting/burning pain & CIPN20 \#5-6 & 0.30 & $2.0 \times 10^{-5}$ & 47 \\
\hline Patient-reported postural hypotension & CIPN20 \#16 & 0.29 & $5.9 \times 10^{-5}$ & 57 \\
\hline Abnormal tendon reflex & TNSc \#6 & 0.28 & $9.6 \times 10^{-5}$ & 101 \\
\hline Patient-reported hand weakness & CIPN20 \#13 & 0.27 & .00017 & 69 \\
\hline Patient-reported cramps & CIPN20 \#7-8 & 0.27 & .00022 & 57 \\
\hline Patient-reported foot drop & CIPN20 \#14 & 0.24 & .001 & 29 \\
\hline Patient-reported hearing difficulty & CIPN20 \#18 & 0.24 & .001 & 44 \\
\hline Patient-reported temperature detection deficit & CIPN20 \#10 & 0.19 & .01 & 19 \\
\hline Abnormal pinprick perception & TNSc \#3 & 0.17 & .02 & 100 \\
\hline Confirmed diabetes diagnosis ${ }^{b}$ & - & 0.09 & .23 & - \\
\hline Patient-reported blurred vision & CIPN20 \#17 & 0.08 & .30 & 34 \\
\hline Sex & - & 0.07 & .32 & - \\
\hline Strength (clinical_manual muscle test) & TNSc \#5 & 0.07 & .36 & 16 \\
\hline Time since completion of neurotoxic cancer treatment ${ }^{b}$ & - & 0.04 & .56 & - \\
\hline
\end{tabular}

Abbreviations: CIPN, chemotherapy-induced peripheral neuropathy; CIPN20, EORTC QLQ-CIPN20 questionnaire; TNSc, Total Neuropathy Score clinical version. Black dashed line denotes critical $\alpha=0.00625$.

aNumber of positive/abnormal responses to each item (out of $\mathrm{N}=190$ patients); not applicable to continuous variables.

${ }^{\mathrm{b}}$ Continuous variable. 


\section{eTable 2. Multiple Regression Analysis of $\mathbf{5}$ Key Correlates of Postural Sway Sumscore and} Patient-Reported Disability

\begin{tabular}{|c|c|c|c|c|}
\hline & Assessment Item & $\mathbf{R}$ & $\boldsymbol{\beta}$ & $P$ Value ${ }^{a}$ \\
\hline Full model & & 0.75 & & $<.001$ \\
\hline Patient-reported leg weakness & CIPN20 \#15 & 0.66 & -0.45 & $<.001$ \\
\hline Patient-reported balance/mobility deficit & CIPN20 \#9 & 0.72 & -0.24 & $<.001$ \\
\hline Age ( $\geq 65$ years) & & 0.74 & 0.18 & .002 \\
\hline Patient-reported numbness/tingling & CIPN20 \#1-4 & 0.75 & 0.20 & .014 \\
\hline Abnormal vibration perception & TNSc \#4 & & & .75 \\
\hline
\end{tabular}

Abbreviations: CIPN, chemotherapy-induced peripheral neuropathy; CIPN20, EORTC QLQ-CIPN20 questionnaire; TNSc, Total Neuropathy Score clinical version ap values correspond to the significance of $\beta$ weights. 